\title{
Retinoblastoma cM1d TNM Finding v7
}

National Cancer Institute

\section{Source}

National Cancer Institute. Retinoblastoma CM1d TNM Finding v7. NCI Thesaurus. Code C88781.

Postchiasmatic CNS lesion(s). (from AJCC 7th Ed.) 